\title{
CORRECTIONS
}

to the paper "Experimental Study of a Diode-Pumped Nd:YAG Slab Laser Amplifier"

Vol. 38, No. 2, pp. 199-203, March 2017

We are sorry about omitting Yanzhong Chen and Jianguo He in the list of authors of the paper Experimental Study of a Diode-Pumped Nd:YAG Slab Laser Amplifier.

The correct reference to the paper is

\section{EXPERIMENTAL STUDY OF A DIODE-PUMPED Nd:YAG SLAB LASER AMPLIFIER}

\author{
Guangyan Guo, ${ }^{1,2}$ Yanzhong Chen, ${ }^{1}$ Jianguo He, ${ }^{1}$ Ye Lang, ${ }^{1,3}$ Weiran Lin, ${ }^{1}$ \\ Zhijun Kang, ${ }^{1}$ Hongbo Zhang, ${ }^{1,4}$ and Zhongwei Fan ${ }^{1,2 *}$ \\ ${ }^{1}$ Academy of Optoelectronics, Chinese Academy of Sciences \\ Beijing, 100094, P. R. China \\ ${ }^{2}$ University of the Chinese Academy of Sciences \\ Beijing, 100049, P. R. China \\ ${ }^{3}$ School of Optoelectronics, Beijing Institute of Technology \\ Beijing, 100081, P. R. China \\ ${ }^{4}$ HarGlo Applied Laser Technology Institute Company, Ltd. \\ Tianjin, 300304, P. R. China \\ ${ }^{*}$ Corresponding author e-mail: fanzhongwei @ aoe.ac.cn
}

